TRANSACTIONS OF THE

AMERICAN MATHEMATICAL SOCIETY

Volume 361, Number 5, May 2009, Pages 2665-2677

S 0002-9947(08)04617-5

Article electronically published on December 4, 2008

\title{
DEPTHS OF MULTIPLIER IDEALS AND INTEGRAL CLOSURE
}

\author{
SEUNGHUN LEE
}

\begin{abstract}
In this note, we study how the depths of multiplier ideals behave under restriction. We also study possible values of the depths of multiplier ideals in the filtrations induced from maximal ideal sheaves. We then use it to give a sufficient condition for the integral closedness of the product of a multiplier ideal and a power of maximal ideal sheaf in the spirit of Huneke.
\end{abstract}

\section{INTRODUCTION}

Let $X$ be a smooth complex variety and $\mathfrak{a}$ be a non-zero ideal sheaf on $X$. Let $\mu: Y \rightarrow X$ be a $\log$ resolution of $\mathfrak{a}$ with $\mu^{*} \mathfrak{a} \cdot \mathcal{O}_{Y}=\mathcal{O}_{Y}(-F)$ where $F$ is a simple normal crossing divisor. Then for any non-negative rational number $\lambda$, one can define the multiplier ideal $\mathcal{J}\left(\mathfrak{a}^{\lambda}\right)=\mathcal{J}\left(X, \mathfrak{a}^{\lambda}\right)$ of $\mathfrak{a}$ with weighting coefficient $\lambda$ as

$$
\mathcal{J}\left(\mathfrak{a}^{\lambda}\right)=\mathcal{J}\left(X, \mathfrak{a}^{\lambda}\right)=\mu_{*} \mathcal{O}_{Y}\left(K_{Y / X}-\lfloor\lambda F\rfloor\right) \subseteq \mathcal{O}_{X}
$$

It does not depend on the choice of the $\log$ resolution $\mu$. We refer to the book [Laz00] of Lazarsfeld for its properties and applications. See also the report EM06] of Ein and Mustaţă on recent developments.

In this note, we will investigate some local properties of multiplier ideals. A recent study of Lazarsfeld and Lee [LL06] on the local syzygies of multiplier ideals shows, for example, that they are very special among all integrally closed ideals. However, less is known about the nature of the multiplier ideal compared to its great success in applications.

We begin with a theorem that shows an interesting behavior of the depth of multiplier ideals under restriction. To state the theorem, we need to introduce a notion which plays a key role in our paper.

Definition 1.1. Let $X$ be a smooth variety. Let $x$ be a closed point of $X$ and $\mathfrak{m}_{x}$ be the ideal sheaf at $x$. Let $\mathfrak{a}$ and $\mathfrak{b}$ be non-zero ideal sheaves on $X$. Let $\lambda$ be a non-negative rational number. Define the length of constancy $c_{x}\left(\mathfrak{b}, \mathfrak{a}^{\lambda}\right)$ of $\mathfrak{a}$ with weighting coefficient $\lambda$, or $\mathfrak{a}^{\lambda}$, relative to $\mathfrak{b}$ at $x$ to be a non-negative integer

$$
\mathrm{c}_{x}\left(\mathfrak{b}, \mathfrak{a}^{\lambda}\right):=\max \left\{n \in \mathbb{Z}_{\geq 0} \mid \mathcal{J}\left(\mathfrak{b}^{n} \cdot \mathfrak{a}^{\lambda}\right)_{x}=\mathcal{J}\left(\mathfrak{a}^{\lambda}\right)_{x}\right\}
$$

if $\mathfrak{b} \subset \mathfrak{m}_{x}$. Otherwise we set $c_{x}\left(\mathfrak{b}, \mathfrak{a}^{\lambda}\right)=\infty$. When $\mathfrak{b}=\mathfrak{m}_{x}$, we also write

$$
\mathrm{c}_{x}\left(\mathfrak{a}^{\lambda}\right)
$$

for $\mathrm{c}_{x}\left(\mathfrak{m}_{x}, \mathfrak{a}^{\lambda}\right)$.

Received by the editors April 6, 2007 and, in revised form, August 18, 2007.

2000 Mathematics Subject Classification. Primary 14E99; Secondary 13C15, 13 B22.

Key words and phrases. Multiplier ideal, depth, integral closure.

This research was supported by R14-2002-007-01001-0. 
In a sense, $\mathfrak{a}^{\lambda}$ becomes more "singular" at $x$ as $c_{x}\left(\mathfrak{a}^{\lambda}\right)$ gets smaller. The length of constancy has an easy upper bound $\operatorname{dim} X-1$ (see Proposition [3.1). However, it seems difficult to calculate them for arbitrary ideals.

The following theorem shows how the lengths of constancy and the depths of multiplier ideals behave under restriction.

Theorem 1.2. Let $X$ be a smooth quasi-projective variety of dimension $d \geq 2$. Let $\mathfrak{a}$ be a non-zero ideal sheaf on $X$ and let $\lambda$ be a non-negative rational number. Let $x$ be a closed point of $X$ and $\mathfrak{m}_{x}$ be the ideal sheaf at $x$.

(1) Suppose that $\mathrm{c}_{x}\left(\mathfrak{a}^{\lambda}\right)>0$. Let $1 \leq n \leq \mathrm{c}_{x}\left(\mathfrak{a}^{\lambda}\right)$. Let $H_{1}, \ldots, H_{n}$ be $n$ general hypersurfaces containing $x$ and let $V=\bigcap_{i=1}^{n} H_{i}$. Then,

$$
\mathrm{c}_{x}\left(\mathfrak{a}^{\lambda}\right)=n+\mathrm{c}_{x}\left(\left(\mathfrak{a}_{V}\right)^{\lambda}\right)
$$

and

$$
\begin{aligned}
\operatorname{depth}_{x, X} \mathcal{J}\left(\mathfrak{a}^{\lambda}\right) & =n+\operatorname{depth}_{x, V} \mathcal{J}\left(\left(\mathfrak{a}_{V}\right)^{\lambda}\right), \\
\operatorname{projdim}_{x, X} \mathcal{J}\left(\mathfrak{a}^{\lambda}\right) & =\operatorname{projdim}_{x, V} \mathcal{J}\left(\left(\mathfrak{a}_{V}\right)^{\lambda}\right),
\end{aligned}
$$

where $\mathfrak{a}_{V}$ is the image of $\mathfrak{a}$ in $\mathcal{O}_{V}$.

(2) Suppose that $\mathrm{c}_{x}\left(\mathfrak{a}^{\lambda}\right)=0$. Let $H$ be a general hypersurface containing $x$. If

$$
\operatorname{depth}_{x, X} \mathcal{J}\left(\mathfrak{a}^{\lambda}\right)>1 \text {, }
$$

then

$$
\operatorname{depth}_{x, H} \mathcal{J}\left(\left(\mathfrak{a}_{H}\right)^{\lambda}\right)=1,
$$

where $\mathfrak{a}_{H}$ is the image of $\mathfrak{a}$ in $\mathcal{O}_{H}$.

Remark 1.3. We do not know of any ideal sheaf $\mathfrak{a} \subseteq \mathcal{O}_{X}$ with $\mathrm{c}_{x}\left(\mathfrak{a}^{\lambda}\right)=0$ such that $\operatorname{depth}_{x, X} \mathcal{J}\left(\mathfrak{a}^{\lambda}\right)=1$ and $\operatorname{depth}_{x, H} \mathcal{J}\left(\left(\mathfrak{a}_{H}\right)^{\lambda}\right) \geq 2$ hold for a general hypersurface $H$ containing $x$.

Our second theorem concerns the depths of multiplier ideals in the following filtration:

$$
\left\{\mathcal{J}\left(\mathfrak{m}_{x}^{n} \cdot \mathfrak{a}^{\lambda}\right)\right\}_{n \geq 0} .
$$

It is easy to see how they behave asymptotically. If $n \geq d$, then $\mathcal{J}\left(\mathfrak{m}_{x}^{n} \cdot \mathfrak{a}^{\lambda}\right)=$ $\mathfrak{m}_{x}^{n-d+1} \cdot \mathcal{J}\left(\mathfrak{m}_{x}^{d-1} \cdot \mathfrak{a}^{\lambda}\right)$ by Theorem 2.2 . Hence, $\mathfrak{m}_{x, X} \in \operatorname{Ass}\left(\mathcal{O}_{x, X} / \mathcal{J}\left(\mathfrak{m}_{x}^{n} \cdot \mathfrak{a}^{\lambda}\right)_{x}\right)$. Thus

$$
\operatorname{depth}_{x, X} \mathcal{J}\left(\mathfrak{m}_{x}^{n} \cdot \mathfrak{a}^{\lambda}\right)=1 .
$$

In fact, depth ${ }_{x, X} \mathcal{J}\left(\mathfrak{a}^{\lambda}\right)$ and 1 are all that we can get from the filtration.

Theorem 1.4. Let $X$ be a smooth quasi-projective variety of dimension $d \geq 2$. Let $\mathfrak{a}$ be a non-zero ideal sheaf on $X$ and let $\lambda$ be a non-negative rational number. Let $x$ be a closed point of $X$ and $\mathfrak{m}_{x}$ be the ideal sheaf at $x$. Then

$$
\operatorname{depth}_{x, X} \mathcal{J}\left(\mathfrak{m}_{x}^{n} \cdot \mathfrak{a}^{\lambda}\right)=\left\{\begin{array}{cl}
\operatorname{depth}_{x, X} \mathcal{J}\left(\mathfrak{a}^{\lambda}\right) & \text { if } 0 \leq n \leq \mathrm{c}_{x}\left(\mathfrak{a}^{\lambda}\right), \\
1 & \text { if } \mathrm{c}_{x}\left(\mathfrak{a}^{\lambda}\right)<n .
\end{array}\right.
$$

We make a couple of remarks about Theorem 1.2 and Theorem 1.4. First, these two results can be extended to the pair $(X, \Delta)$ where $X$ is a normal Cohen-Macaulay variety and $K_{X}+\Delta$ is a $\mathbb{Q}$-Cartier $\mathbb{Q}$-divisor on $X$. Second, since reduction to prime characteristic preserves depth (cf. Theorem 2.3.5 in HH99]) and the multiplier ideal reduces generically to the test ideals of Hara and Yoshida [HY03] and Takagi 
Tak04, one obtains analogues of these two results in large characteristics. Whether this holds for arbitrary characteristic is unknown.

We now turn to the integral closedness. Zariski (cf. [ZS75]) showed that the product of any two integrally closed ideals in a regular local ring of dimension two is again integrally closed. One special but important case is when one of the ideals is the maximal ideal. Neither of these cases remain true if the dimension is greater than two. See the examples in $\S 3$ in Hun86]. Our motivation is the following theorem of Huneke.

Theorem 1.5 (Theorem 3.5 in Hun86). Let I be an ideal in a regular local ring $(R, \mathfrak{m})$ of dimension $\leq 3$ with $R / \mathfrak{m}$ infinite. Suppose for a general $x \in \mathfrak{m} / \mathfrak{m}^{2}$ that $(I, x) /(x) \subset R /(x)$ is integrally closed. Then $\overline{\mathfrak{m}^{n} \cdot I}=\mathfrak{m}^{n} \cdot \bar{I}$ for all $n \geq 1$. Here $\bar{J}$ denotes the integral closure of an ideal $J \subset R$.

The proof of Huneke's theorem proceeds by induction on dimension, and the key role is played by the theorem of Zariski mentioned above. This is why the proof cannot go beyond dimension three. However, multiplier ideals are integrally closed, and if we restrict to them instead of all integrally closed ideals, Huneke's arguments can provide us with a sufficient condition in terms of depth that works for all dimensions.

Theorem 1.6. Let $X$ be a smooth affine variety of dimension $d \geq 2$. Let $\mathfrak{a}$ be an ideal sheaf on $X$ and let $\lambda$ be a non-negative rational number. Let $x$ be a closed point of $X$ and $\mathfrak{m}_{x}$ be the ideal sheaf at $x$. Suppose that

$$
\operatorname{depth}_{x, X} \mathcal{J}\left(\mathfrak{m}_{x}^{d-2} \cdot \mathfrak{a}^{\lambda}\right) \geq 2 .
$$

Then, for any non-negative integer $k$, the ideal sheaf

$$
\mathfrak{m}_{x}^{k} \cdot \mathcal{J}\left(\mathfrak{a}^{\lambda}\right)
$$

is integrally closed.

The above theorem is a consequence of Theorem 1.4 and the following theorem. Note that, by the Skoda theorem (Theorem 2.2$), \mathfrak{m}_{x}^{k} \cdot \mathcal{J}\left(\mathfrak{m}_{x}^{n} \cdot \mathfrak{a}^{\lambda}\right.$ ) is integrally closed if $n \geq d-1$. Hence the essential part is the case of $n=d-2$ and $d \geq 3$, which is what we need to prove Theorem 1.6.

Theorem 1.7. Let $X$ be a smooth affine variety of dimension $d \geq 2$. Let $\mathfrak{a}$ be an ideal sheaf on $X$ and let $\lambda$ be a non-negative rational number. Let $x$ be a closed point of $X$ and $\mathfrak{m}_{x}$ be the ideal sheaf at $x$. Let $k$ and $n$ be two non-negative integers. If $n \geq d-2$, the ideal sheaf

$$
\mathfrak{m}_{x}^{k} \cdot \mathcal{J}\left(\mathfrak{m}_{x}^{n} \cdot \mathfrak{a}^{\lambda}\right)
$$

is integrally closed.

For a result with a different flavor, we have the following

Theorem 1.8. Let $X$ be a smooth affine variety of dimension $d \geq 2$. Let $\mathfrak{a}$ be an ideal sheaf on $X$ and let $\lambda$ be a non-negative rational number. Let $x$ be a closed point of $X$ and $\mathfrak{m}_{x}$ be the ideal sheaf at $x$. Let $1 \leq c \leq d-1$. Let $H_{1}, \ldots, H_{c}$ be c general hypersurfaces containing $x$ and let $V=\bigcap_{i=1}^{c} H_{i}$. Then for any integer $k \geq c$, the ideal sheaf

$$
\mathcal{I}_{V} \otimes \mathcal{J}\left(\mathfrak{m}_{x}^{k-1} \cdot \mathfrak{a}^{\lambda}\right)
$$

is integrally closed, where $\mathcal{I}_{V}$ is the ideal sheaf along $V$. 
Remark 1.9. It would be interesting to know if a similar statement holds for arbitrary integrally closed ideals.

Question 1.10. Let $X$ be a smooth affine variety of dimension $d \geq 2$. Let $\mathfrak{a}$ be an ideal sheaf on $X$. Let $\lambda$ be a non-negative rational number. Let $x$ be a closed point of $X$ and $\mathfrak{m}_{x}$ be the ideal sheaf at $x$. Is the ideal sheaf

$$
\mathfrak{m}_{x}^{k} \cdot \mathcal{J}\left(\mathfrak{a}^{\lambda}\right)
$$

integrally closed for any $k \geq 0$ ?

We work over the field $\mathbb{C}$ of complex numbers.

\section{RevieW of MULTiPlier ideAls, DePths AND INTEGRAL ClOSURES}

2.1. Multiplier ideals. The following proposition is well-known.

Proposition 2.1. Let $\mathfrak{a}$ and $\mathfrak{b}$ be ideal sheaves on a smooth variety $X$. Then for any non-negative rational number $\lambda$,

$$
\mathfrak{a} \cdot \mathcal{J}\left(\mathfrak{b}^{\lambda}\right) \subset \mathcal{J}\left(\mathfrak{a} \cdot \mathfrak{b}^{\lambda}\right) .
$$

Theorem 2.2 (Skoda's theorem; Theorem 9.6.21 in [Laz00]). Let $\mathfrak{a}$ and $\mathfrak{b}$ be ideal sheaves on a smooth variety $X$ of dimension $d$ and let $\lambda$ be a non-negative rational number. Then, for any integer $m \geq d$,

$$
\mathcal{J}\left(\mathfrak{a}^{m} \cdot \mathfrak{b}^{\lambda}\right)=\mathfrak{a}^{m-d+1} \cdot \mathcal{J}\left(\mathfrak{a}^{d-1} \cdot \mathfrak{b}^{\lambda}\right) .
$$

The following is a refined version of the restriction theorem (cf. Theorem 9.5.1 in [Laz00]). We state only the case of restriction to hypersurfaces.

Theorem 2.3 (Theorem 1.6 in Lee06). Let $X$ be a smooth quasi-projective variety of dimension $d \geq 2$. Let $\mathfrak{a}$ be an ideal sheaf on $X$ and let $\lambda$ be a non-negative rational number. Let $x$ be a closed point of $X$ and $\mathfrak{m}_{x}$ be the ideal sheaf at $x$. Let $H$ be a general hypersurface containing $x$. Then we have the following commutative diagram:

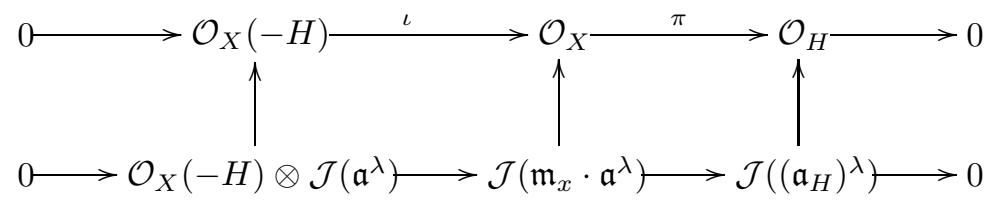

where $\mathfrak{a}_{H} \subset \mathcal{O}_{H}$ is the image of $\mathfrak{a}$ in $\mathcal{O}_{H}$, ८ and vertical maps are the natural inclusions and $\pi$ is the natural surjection.

The proof of the following theorem is similar to that of Theorem 1.6 in Lee06]. So we omit the proof.

Theorem 2.4. Let $X$ be a smooth quasi-projective variety of dimension $d \geq 2$. Let $\mathfrak{a}$ be an ideal sheaf on $X$ and let $\lambda$ be a non-negative rational number. Let $Z$ be a smooth subvariety of $X$ with $\operatorname{codim} Z \geq 2$ and $\mathcal{I}_{Z}$ be the ideal sheaf along $Z$. Let $1 \leq c \leq \operatorname{codim} Z-1$ and let $H_{1}, \ldots, H_{c}$ be $c$ general hypersurfaces containing $Z$. Let $V=\bigcap_{i=1}^{c} H_{i}$. Then for any integer $k \geq c$, we have the following commutative 
diagram:

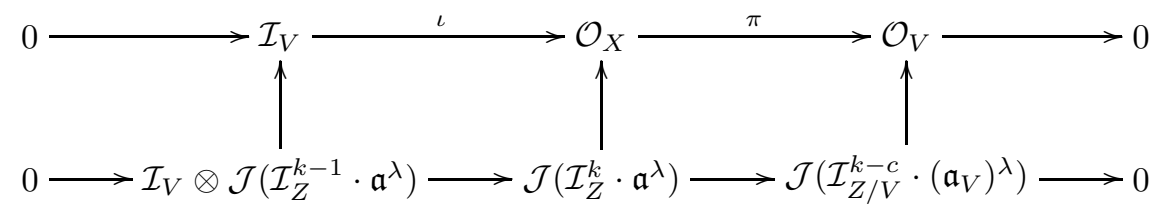

where $\mathcal{I}_{V}$ is the ideal sheaf along $V, \mathfrak{a}_{V} \subset \mathcal{O}_{V}$ is the image of $\mathfrak{a}$ in $\mathcal{O}_{V}, \mathcal{I}_{Z / V} \subset \mathcal{O}_{V}$ is the image of $\mathcal{I}_{Z}$ in $\mathcal{O}_{V}$, $\iota$ and vertical maps are the natural inclusions and $\pi$ is the natural surjection.

Corollary 2.5. With the same situation as in Theorem 2.4,

$$
\mathcal{I}_{V} \otimes \mathcal{J}\left(\mathcal{I}_{Z}^{k-1} \cdot \mathfrak{a}^{\lambda}\right)=\mathcal{I}_{V} \cap \mathcal{J}\left(\mathcal{I}_{Z}^{k} \cdot \mathfrak{a}^{\lambda}\right) .
$$

2.2. Depths, dimensions, and projective dimensions. First, we recall the depth.

Definition 2.6. Let $R$ be a Noetherian commutative ring with unity. Let $I$ be an ideal of $R$ and let $M$ be a finite $R$-module with $I M \neq M$. A sequence of elements $a_{1}, \ldots, a_{n}$ in $I$ is said to be a $M$-regular sequence in $I$ if for all $i=0, \ldots, n-1$,

$$
\left(\left(a_{1}, \ldots, a_{i}\right) M: a_{i+1}\right) \cap M=\left(a_{1}, \ldots, a_{i}\right) M .
$$

Here $\left(a_{1}, \ldots, a_{i}\right) M=0$ for $i=0$ by convention. The length of a (or any) maximal $M$-regular sequence in $I$ is called the depth of $M$ in $I$ and denoted by

$$
\operatorname{depth}(I, M) \text {. }
$$

Proposition 2.7 (cf. Proposition 18.4 in [Eis99]). Let $R$ be a Noetherian commutative ring with unity. Let $I$ be an ideal of $R$ and let $M$ be a finite $R$-module with $I M \neq M$. Then

$$
\operatorname{depth}(I, M)=\min \left\{n \mid \operatorname{Ext}^{n}(R / I, M) \neq 0\right\} .
$$

The next proposition is a consequence of Proposition 2.7

Proposition 2.8 (cf. Corollary 18.6 in [Eis99]). Let $R$ be a Noetherian commutative ring with unity and let $I$ be an ideal of $R$. Let

$$
0 \rightarrow M^{\prime} \rightarrow M \rightarrow M^{\prime \prime} \rightarrow 0
$$

be an exact sequence of finite $R$-modules with $I M^{\prime} \neq M^{\prime}, I M \neq M$, and $I M^{\prime \prime} \neq$ $M^{\prime \prime}$. Then one of the following three conditions must hold:

(1) $\operatorname{depth}\left(I, M^{\prime}\right)=\operatorname{depth}(I, M) \leq \operatorname{depth}\left(I, M^{\prime \prime}\right)$.

(2) $\operatorname{depth}(I, M)=\operatorname{depth}\left(I, M^{\prime \prime}\right)<\operatorname{depth}\left(I, M^{\prime}\right)$.

(3) $\operatorname{depth}\left(I, M^{\prime \prime}\right)+1=\operatorname{depth}\left(I, M^{\prime}\right) \leq \operatorname{depth}(I, M)$.

Definition 2.9. Let $X$ be a variety. Let $\mathcal{F}$ be a sheaf of the $\mathcal{O}_{X}$-module and let $x$ be a closed point of $X$. Then we define the depth $\operatorname{depth}_{x} \mathcal{F}$ of $\mathcal{F}$ at $x$ as

$$
\operatorname{depth}_{x, X} \mathcal{F}:=\operatorname{depth}\left(\mathfrak{m}_{x, X}, \mathcal{F}_{x}\right),
$$

where $\left(\mathfrak{m}_{x, X}, \mathcal{O}_{x, X}\right)$ is the local ring at $x$ and $\mathcal{F}_{x}$ is the stalk of $\mathcal{F}$ at $x$.

Proposition 2.10 (cf. Proposition 18.2 in Eis99]). Let $R$ be a Noetherian commutative ring with unity. Let $M$ be a finite $R$-module and let $I$ be an ideal of $R$ containing $\operatorname{Ann}(M)$. Then

$$
\operatorname{depth}(I, M) \leq \operatorname{dim} M .
$$


Proposition 2.11. Let $X$ be a smooth variety. Let $x$ be a closed point of $X$ and $\mathfrak{m}_{x}$ be the ideal sheaf at $x$. Let $\mathfrak{a}$ and $\mathfrak{b}$ be non-zero ideal sheaves on $X$ and let $\lambda$ be a non-negative rational number. Suppose $\mathfrak{b} \subset \mathfrak{m}_{x}$. If $\mathcal{J}\left(\mathfrak{b} \cdot \mathfrak{a}^{\lambda}\right) \varsubsetneqq \mathcal{J}\left(\mathfrak{a}^{\lambda}\right)$, then

$$
\operatorname{depth}_{x, X} \mathcal{J}\left(\mathfrak{a}^{\lambda}\right) / \mathcal{J}\left(\mathfrak{b} \cdot \mathfrak{a}^{\lambda}\right) \leq \operatorname{dim} \mathcal{O}_{x, X} / \mathfrak{b}_{x},
$$

where $\mathcal{O}_{x, X}$ and $\mathfrak{b}_{x}$ are the stalks of $\mathcal{O}_{X}$ and $\mathfrak{b}$ at $x$, respectively.

Proof. From Proposition 2.1.

$$
\mathfrak{b} \cdot \mathcal{J}\left(\mathfrak{a}^{\lambda}\right) \subset \mathcal{J}\left(\mathfrak{b} \cdot \mathfrak{a}^{\lambda}\right) .
$$

Then

$$
\mathfrak{b} \subset\left(0: \mathcal{J}\left(\mathfrak{a}^{\lambda}\right) / \mathcal{J}\left(\mathfrak{b} \cdot \mathfrak{a}^{\lambda}\right)\right) .
$$

Hence from Proposition 2.10

$$
\begin{aligned}
\operatorname{depth}_{x, X} \mathcal{J}\left(\mathfrak{a}^{\lambda}\right) / \mathcal{J}\left(\mathfrak{b} \cdot \mathfrak{a}^{\lambda}\right) & \leq \operatorname{dim}\left(\mathcal{J}\left(\mathfrak{a}^{\lambda}\right) / \mathcal{J}\left(\mathfrak{b} \cdot \mathfrak{a}^{\lambda}\right)\right)_{x} \\
& =\operatorname{dim} \mathcal{O}_{x, X} /\left(0:\left(\mathcal{J}\left(\mathfrak{a}^{\lambda}\right) / \mathcal{J}\left(\mathfrak{b} \cdot \mathfrak{a}^{\lambda}\right)\right)_{x}\right) \\
& \leq \operatorname{dim} \mathcal{O}_{x, X} / \mathfrak{b}_{x} .
\end{aligned}
$$

Finally, we recall the projective dimension.

Definition 2.12. Let $R$ be a commutative ring with unity and let $M$ be a $R$ module. The projective dimension

$$
\operatorname{projdim}_{R} M
$$

is the minimum of the lengths of projective resolutions of $M$. It is defined to be $\infty$ if $M$ has no finite projective resolution.

Theorem 2.13 (cf. Theorem 19.9 in Eis99]). Let $(R, \mathfrak{m})$ be a Noetherian commutative local ring with unity and let $M$ be a finitely generated $R$-module of finite projective dimension. Then

$$
\operatorname{projdim}_{R} M=\operatorname{depth}(\mathfrak{m}, R)-\operatorname{depth}(\mathfrak{m}, M) .
$$

Definition 2.14. Let $X$ be a variety. Let $\mathcal{F}$ be a sheaf of the $\mathcal{O}_{X}$-module and let $x$ be a closed point of $X$. Then we define the projective $\operatorname{dimension}_{\operatorname{projdim}_{x}} \mathcal{F}$ of $\mathcal{F}$ at $x$ as

$$
\operatorname{projdim}_{x, X} \mathcal{F}:=\operatorname{projdim}_{\mathcal{O}_{x, X}} \mathcal{F}_{x},
$$

where $\left(\mathfrak{m}_{x, X}, \mathcal{O}_{x, X}\right)$ is the local ring of $X$ at $x$ and $\mathcal{F}_{x}$ is the stalk of $\mathcal{F}$ at $x$.

\section{3. ( $N, M)$-regular sequences.}

Definition 2.15. Let $R$ be a commutative ring with unity. Let $M$ be a $R$-module and let $N$ be a submodule of $M$. A sequence of elements $a_{1}, \ldots, a_{n}$ in $R$ is said to be an $(N, M)$-regular sequence if

(1) $\left(a_{1}, \ldots, a_{n}\right) M \subset N$,

(2) for all $i=0, \ldots, n-1$,

$$
\left(\left(a_{1}, \ldots, a_{i}\right) M: a_{i+1}\right) \cap N=\left(a_{1}, \ldots, a_{i}\right) M .
$$

Here $\left(a_{1}, \ldots, a_{i}\right) M=0$ for $i=0$ by convention.

As usual, we identify a coherent sheaf on an affine variety with the module of its global sections. 
Theorem 2.16 (Theorem 1.4 in [Lee06]). Let $X$ be a smooth affine variety of dimension $d \geq 2$. Let $\mathfrak{a}$ be an ideal sheaf on $X$ and let $\lambda$ be a non-negative rational number. Let $x$ be a closed point in $X$ and $\mathfrak{m}_{x}$ be the ideal sheaf at $x$. Let $k$ and $n$ be non-negative integers with $1 \leq n \leq d$ and $k \geq n-2$. Then for a general choice $a_{1}, \ldots, a_{n}$ of elements in $\mathfrak{m}_{x}$

(1) $\left\{a_{1}, \ldots, a_{n}\right\}$ is a $\left(\mathcal{J}\left(\mathfrak{m}_{x}^{k+1} \cdot \mathfrak{a}^{\lambda}\right), \mathcal{J}\left(\mathfrak{m}_{x}^{k} \cdot \mathfrak{a}^{\lambda}\right)\right)$-regular sequence, hence

(2) the natural map

$$
H_{p}\left(K_{\bullet}\left(a_{1}, \ldots, a_{n}\right) \otimes \mathcal{J}\left(\mathfrak{m}_{x}^{k+1} \cdot \mathfrak{a}^{\lambda}\right) \rightarrow K_{\bullet}\left(a_{1}, \ldots, a_{n}\right) \otimes \mathcal{J}\left(\mathfrak{m}_{x}^{k} \cdot \mathfrak{a}^{\lambda}\right)\right)
$$

vanishes for any $p>0$.

2.4. Integral closure. The following proposition slightly generalizes the key step of the proof of Theorem 3.5 in Hun86] in characteristic zero.

Proposition 2.17. Let $X$ be a smooth affine variety. Let $\mathfrak{a}$ and $\mathfrak{b}$ be non-zero ideal sheaves on $X$ and let $x \in \mathfrak{a}$ be a general element. We let $H:=\operatorname{div}(x)$. If $\mathfrak{a}, \mathfrak{b}$ and $(\mathfrak{a} \cdot \mathfrak{b}) \cdot \mathcal{O}_{H}$ are integrally closed, then

$$
\mathfrak{a} \cdot \mathfrak{b}=\overline{\mathfrak{a} \cdot \mathfrak{b}} .
$$

Theorem 2.18 (Theorem 7.1 in Lip69). Let $\mathfrak{a}$ and $\mathfrak{b}$ be ideal sheaves on a smooth surface $S$. Then

$$
\overline{\mathfrak{a}} \overline{\mathfrak{b}}=\overline{\mathfrak{a} \mathfrak{b}} .
$$

Proposition 2.19 (Corollary 9.6.13 in Laz00]). Let $\mathfrak{a}$ be an ideal on a smooth variety $X$. Then for any non-negative rational number $\lambda$, the multiplier ideal $\mathcal{J}\left(\mathfrak{a}^{\lambda}\right)$ is integrally closed.

\section{Depths of Multiplier ideals}

3.1. On the length of constancy. From the Skoda theorem, we obtain the following upper bound for the length of constancy.

Proposition 3.1. Let $X$ be a smooth variety of dimension $d$. Let $x$ be a closed point of $X$ and $\mathfrak{m}_{x}$ be the ideal sheaf at $x$. Let $\mathfrak{a}$ and $\mathfrak{b}$ be non-zero ideal sheaves on $X$. Let $\lambda$ be a non-negative rational number. Suppose that $\mathfrak{b} \subset \mathfrak{m}_{x}$. Then

$$
\mathrm{c}_{x}\left(\mathfrak{b}, \mathfrak{a}^{\lambda}\right) \leq d-1 .
$$

Proof. We may assume that $X$ is an affine variety. Let $n \geq d$, and suppose that $\mathcal{J}\left(\mathfrak{b}^{n}, \mathfrak{a}^{\lambda}\right)=\mathcal{J}\left(\mathfrak{a}^{\lambda}\right)$. By Theorem 2.2.

$$
\mathfrak{b}^{n-d+1} \cdot \mathcal{J}\left(\mathfrak{b}^{d-1} \cdot \mathfrak{a}^{\lambda}\right)=\mathcal{J}\left(\mathfrak{b}^{n} \cdot \mathfrak{a}^{\lambda}\right) .
$$

Hence

$$
\mathfrak{b}^{n-d+1} \cdot \mathcal{J}\left(\mathfrak{a}^{\lambda}\right)=\mathcal{J}\left(\mathfrak{a}^{\lambda}\right)
$$

By the Cayley-Hamilton Theorem (cf. Theorem 4.3 in [Eis99]), there exists $r \in$ $\mathfrak{b}^{n-d+1}$ such that $(1-r) \cdot \mathcal{J}\left(\mathfrak{a}^{\lambda}\right)=0$. Since $X$ is irreducible and reduced, it implies that $\mathcal{J}\left(\mathfrak{a}^{\lambda}\right)=0$, a contradiction.

The following example shows that every value between 0 and $\operatorname{dim} X-1$ can be obtained as the length of constancy.

Example 3.2. Let $X$ be a smooth variety of dimension $d$. Let $x$ be a closed point of $X$ and $\mathfrak{m}_{x}$ be the ideal sheaf at $x$. Let $k$ be a non-negative integer. Then

$$
\mathrm{c}_{x}\left(\mathfrak{m}_{x}^{k}\right)=\max \{0, d-1-k\} .
$$


It seems difficult to calculate the length of constancy for arbitrary ideals. However, we will give one sufficient condition for $c_{x}\left(\mathfrak{a}^{\lambda}\right)=0$ in Corollary 3.8.

The following proposition follows immediately from the definition of the length of constancy.

Proposition 3.3. Let $X$ be a smooth variety. Let $x$ be a closed point of $X$ and $\mathfrak{m}_{x}$ be the ideal sheaf at $x$. Let $\mathfrak{a}$ and $\mathfrak{b}$ be non-zero ideal sheaves on $X$. Let $\lambda$ be a non-negative rational number. Suppose that $\mathfrak{b} \subset \mathfrak{m}_{x}$. Then

$$
\mathrm{c}_{x}\left(\mathfrak{b}, \mathfrak{b}^{n} \cdot \mathfrak{a}^{\lambda}\right)=\mathrm{c}_{x}\left(\mathfrak{b}, \mathfrak{a}^{\lambda}\right)-n
$$

for any $0 \leq n \leq \mathrm{c}_{x}\left(\mathfrak{b}, \mathfrak{a}^{\lambda}\right)$.

The following theorem shows how the length of constancy behaves under restriction.

Theorem 3.4. Let $X$ be a smooth quasi-projective variety of dimension $d \geq 2$. Let $\mathfrak{a}$ be a non-zero ideal sheaf on $X$ and let $\lambda$ be a non-negative rational number. Let $x$ be a closed point of $X$ and let $H$ be a general hypersurface containing $x$. If $\mathrm{c}_{x}\left(\mathfrak{a}^{\lambda}\right)>0$, then

$$
\mathrm{c}_{x}\left(\mathfrak{a}^{\lambda}\right)=\mathrm{c}_{x}\left(\left(\mathfrak{a}_{H}\right)^{\lambda}\right)+1,
$$

where $\mathfrak{a}_{H}$ is the image of $\mathfrak{a}$ in $\mathcal{O}_{H}$.

Proof. Let $\mathfrak{m}_{x}$ be the ideal sheaf at $x$ and $\mathfrak{m}_{x / H}$ be its image in $\mathcal{O}_{H}$. Let $\mathrm{c}:=\mathrm{c}_{x}\left(\mathfrak{a}^{\lambda}\right)$. From Theorem 2.3 , we have the following commutative diagram:

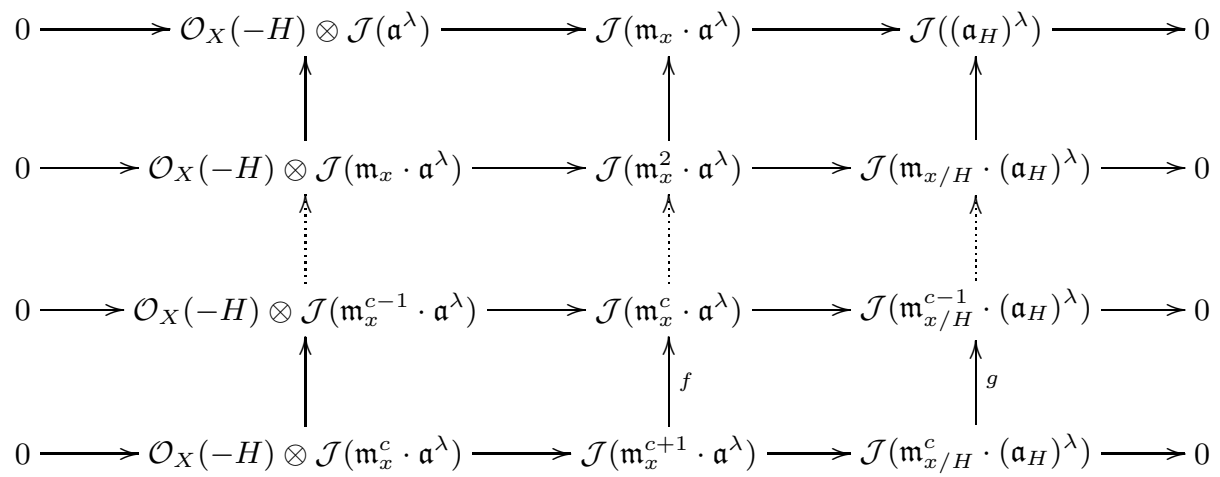

where all vertical maps in the first column and the second column are isomorphisms except $f$ by our assumption. Hence all vertical maps in the third column are isomorphisms except $g$.

Remark 3.5. We do not know any ideal sheaf $\mathfrak{a} \subseteq \mathcal{O}_{X}$ with $\mathrm{c}_{x}\left(\mathfrak{a}^{\lambda}\right)=0$ such that $\mathrm{c}_{x}\left(\left(\mathfrak{a}_{H}\right)^{\lambda}\right)>0$ holds for a general hypersurface $H$ containing $x$.

3.2. Depths of multiplier ideals. Now we are ready to prove the two results on depth stated in the Introduction. First, we record the following simple proposition.

Proposition 3.6. Let $X$ be a smooth quasi-projective variety of dimension $\geq 2$. Let $\mathfrak{a}$ be a non-zero ideal sheaf on $X$ and let $\lambda$ be a non-negative rational number. Let $x$ be a closed point of $X$ and $\mathfrak{m}_{x}$ be the ideal sheaf at $x$. Let $\mathrm{c}:=\mathrm{c}_{x}\left(\mathfrak{a}^{\lambda}\right)$. Then

$$
\operatorname{depth}_{x, X} \mathcal{J}\left(\mathfrak{m}_{x}^{c+1} \cdot \mathfrak{a}^{\lambda}\right)=1 .
$$


Proof. By Proposition 3.3, we may assume that $\mathrm{c}=0$. Consider

$$
0 \longrightarrow \mathcal{J}\left(\mathfrak{m}_{x} \cdot \mathfrak{a}^{\lambda}\right) \longrightarrow \mathcal{J}\left(\mathfrak{a}^{\lambda}\right) \longrightarrow \mathcal{J}\left(\mathfrak{a}^{\lambda}\right) / \mathcal{J}\left(\mathfrak{m}_{x} \cdot \mathfrak{a}^{\lambda}\right) \longrightarrow 0
$$

Note that, since $\mathrm{c}=0, \mathcal{J}\left(\mathfrak{a}^{\lambda}\right) / \mathcal{J}\left(\mathfrak{m}_{x} \cdot \mathfrak{a}^{\lambda}\right) \neq 0$. From Proposition 2.11

$$
\operatorname{depth}_{x, X} \mathcal{J}\left(\mathfrak{a}^{\lambda}\right) / \mathcal{J}\left(\mathfrak{m}_{x} \cdot \mathfrak{a}^{\lambda}\right)=0 .
$$

Since $\mathcal{O}_{x, X}$ is an integral domain, $\operatorname{depth}_{x, X} \mathcal{J}\left(\mathfrak{m}_{x} \cdot \mathfrak{a}^{\lambda}\right) \geq 1$ and $\operatorname{depth}_{x, X} \mathcal{J}\left(\mathfrak{a}^{\lambda}\right) \geq 1$. Thus Proposition 2.8(3) implies that $\operatorname{depth}_{x, X} \mathcal{J}\left(\mathfrak{m}_{x} \cdot \mathfrak{a}^{\lambda}\right)=1$.

Next we prove Theorem 1.2

Proof of Theorem 1.2. (1) The first statement follows from Theorem 3.4. The last statement follows from the second and from Theorem 2.13. To prove the second statement, it will be enough to show that if $\mathrm{c}_{x}\left(\mathfrak{a}^{\lambda}\right) \geq 1$, then

$$
\operatorname{depth}_{x, X} \mathcal{J}\left(\mathfrak{a}^{\lambda}\right)=1+\operatorname{depth}_{x, H} \mathcal{J}\left(\left(\mathfrak{a}_{H}\right)^{\lambda}\right),
$$

where $H$ is a general hypersurface containing $x$. From Theorem 2.3, we have

$$
0 \longrightarrow \mathcal{O}_{X}(-H) \otimes \mathcal{J}\left(\mathfrak{a}^{\lambda}\right) \longrightarrow \mathcal{J}\left(\mathfrak{m}_{x} \cdot \mathfrak{a}^{\lambda}\right) \longrightarrow \mathcal{J}\left(\left(\mathfrak{a}_{H}\right)^{\lambda}\right) \longrightarrow 0,
$$

where $H$ is a general hypersurface containing $x$. Since $\mathcal{O}_{x, X}$ is an integral domain, a local equation of $\mathcal{O}_{X}(-H)$ at $x$ is $\mathcal{J}\left(\mathfrak{a}^{\lambda}\right)_{x}$-regular. Then, since $\mathcal{J}\left(\mathfrak{a}^{\lambda}\right)=\mathcal{J}\left(\mathfrak{m}_{x} \cdot \mathfrak{a}^{\lambda}\right)$ by our assumption, from the above short exact sequence we obtain

$$
\begin{aligned}
\operatorname{depth}_{x, H} \mathcal{J}\left(\left(\mathfrak{a}_{H}\right)^{\lambda}\right) & =\operatorname{depth}_{x, X} \mathcal{J}\left(\left(\mathfrak{a}_{H}\right)^{\lambda}\right) \\
& =\operatorname{depth}_{x, X} \mathcal{J}\left(\mathfrak{a}^{\lambda}\right) / \mathcal{O}_{X}(-H) \cdot \mathcal{J}\left(\mathfrak{a}^{\lambda}\right) \\
& =\operatorname{depth}_{x, X} \mathcal{J}\left(\mathfrak{a}^{\lambda}\right)-1
\end{aligned}
$$

(2) Again, we consider

$$
0 \longrightarrow \mathcal{O}_{X}(-H) \otimes \mathcal{J}\left(\mathfrak{a}^{\lambda}\right) \longrightarrow \mathcal{J}\left(\mathfrak{m}_{x} \cdot \mathfrak{a}^{\lambda}\right) \longrightarrow \mathcal{J}\left(\left(\mathfrak{a}_{H}\right)^{\lambda}\right) \longrightarrow 0,
$$

where $H$ is a general hypersurface containing $x$. From Proposition 3.6 and $\mathrm{c}_{x}\left(\mathfrak{a}^{\lambda}\right)=$ 0 , we have

$$
\operatorname{depth}_{x, X} \mathcal{J}\left(\mathfrak{m}_{x} \cdot \mathfrak{a}^{\lambda}\right)=1 .
$$

Since $\operatorname{depth}_{x, X} \mathcal{J}\left(\mathfrak{a}^{\lambda}\right)>1$ by our assumption, Proposition 2.8(2) implies that

$$
\operatorname{depth}_{x, H} \mathcal{J}\left(\left(\mathfrak{a}_{H}\right)^{\lambda}\right)=\operatorname{depth}_{x, X} \mathcal{J}\left(\left(\mathfrak{a}_{H}\right)^{\lambda}\right)=1 .
$$

In Theorem 1.2(2), it can happen that both $\mathcal{J}(\mathfrak{a})$ and $\mathcal{J}\left(\mathfrak{a}_{H}\right)$ have depth one as the following simple example shows.

Example 3.7. Let $X$ be a smooth variety of dimension $d \geq 2$. Let $x$ be a closed point of $X$ and $\mathfrak{m}_{x}$ be the ideal sheaf at $x$. Let $H$ be a general hypersurface containing $x$. Let $\mathfrak{a}=\mathfrak{m}_{x}^{d}$. Then $\mathcal{J}(\mathfrak{a})=\mathfrak{m}_{x}$ and $\mathcal{J}\left(\mathfrak{a}_{H}\right)=\mathfrak{m}_{x / H}^{2}$, where $\mathfrak{m}_{x / H}$ is the image of $\mathfrak{m}_{x}$ in $\mathcal{O}_{H}$. Thus

$$
\operatorname{depth}_{x, X} \mathcal{J}(\mathfrak{a})=1=\operatorname{depth}_{x, H} \mathcal{J}\left(\mathfrak{a}_{H}\right) .
$$


It is of some interest to know when

$$
\operatorname{depth}_{x, X} \mathcal{J}\left(\mathfrak{a}^{\lambda}\right)=1
$$

holds. By the Auslander-Buchsbaum theorem (Theorem 2.13), it is equivalent to

$$
\operatorname{projdim}_{x, X} \mathcal{O}_{X} / \mathcal{J}\left(\mathfrak{a}^{\lambda}\right)=\operatorname{dim} X,
$$

i.e. the length of the minimal free resolution of $\left(\mathcal{O}_{X} / \mathcal{J}\left(\mathfrak{a}^{\lambda}\right)\right)_{x}$ has the maximal possible value $\operatorname{dim} X$. Theorem 1.2(1) provides us with the following necessary condition.

Corollary 3.8. Let $X$ be a smooth quasi-projective variety of dimension $\geq 2$. Let $\mathfrak{a}$ be a non-zero ideal sheaf on $X$. Let $\lambda$ be a non-negative rational number. Let $x$ be a closed point of $X$. Then

$$
\operatorname{depth}_{x, X} \mathcal{J}\left(\mathfrak{a}^{\lambda}\right)>\mathrm{c}_{x}\left(\mathfrak{a}^{\lambda}\right) .
$$

In particular, if $\operatorname{depth}_{x, X} \mathcal{J}\left(\mathfrak{a}^{\lambda}\right)=1$, then

$$
\mathrm{c}_{x}\left(\mathfrak{a}^{\lambda}\right)=0 \text {. }
$$

Remark 3.9. Proposition 3.3. Proposition 3.6 and Corollary 3.8 imply that, as a function of $n, \mathrm{c}_{x}\left(\mathfrak{m}_{x}^{n} \cdot \mathfrak{a}^{\lambda}\right)$ is a decreasing function converging to zero.

The following example shows that the converse of the second statement of Corollary 3.8 does not hold.

Example 3.10. Let $X=\mathbb{C}^{3}$. Let $x=(0,0,0)$ and $\mathfrak{m}_{x}=\left(x_{1}, x_{2}, x_{3}\right)$. Let $\mathfrak{b}=$ $\left(x_{1}, x_{2}\right)$ and $\mathfrak{a}=\mathfrak{m}_{x} \cdot \mathfrak{b}^{2}$. Let $f: Y \rightarrow X$ be the blowing up of $X$ at $\mathfrak{m}_{x}$ with the exceptional divisor $E$, and let $g: Z \rightarrow Y$ be the blowing up of $\mathfrak{b} \cdot \mathcal{O}_{Y}$ with the exceptional divisor $F$. We keep the same notation for the proper transform of $E$ in $Z$. Then

$$
\mathfrak{a} \cdot \mathcal{O}_{Z}=-3 E-2 F
$$

and

$$
K_{Z / X}=2 E+F
$$

Hence

and

$$
\mathcal{J}(\mathfrak{a})=(f \circ g)_{*} \mathcal{O}_{Z}(-E-F)=\mathfrak{b}
$$

$$
\mathcal{J}\left(\mathfrak{m}_{x} \cdot \mathfrak{a}\right)=(f \circ g)_{*} \mathcal{O}_{Z}(-2 E-F)=\mathfrak{m}_{x} \cdot \mathfrak{b} .
$$

Thus $c_{x}(\mathfrak{a})=0$. On the other hand, by Theorem 2.13 .

$$
\operatorname{depth}_{x, X} \mathcal{J}(\mathfrak{a})=\operatorname{depth}_{x, X} \mathfrak{b}=\operatorname{depth}_{x, X}\left(\mathcal{O}_{X} / \mathfrak{b}\right)+1=2 .
$$

But we can measure the obstruction using the following proposition.

Proposition 3.11. Let $X$ be a smooth quasi-projective variety of dimension $d \geq 2$. Let $\mathfrak{a}$ be a non-zero ideal sheaf on $X$ and let $\lambda$ be a non-negative rational number. Let $x$ be a closed point of $X$ and $\mathfrak{m}_{x}$ be the ideal sheaf at $x$. Let $a_{1}, a_{2} \in \mathfrak{m}_{x}$ be general elements so that $a_{1}, a_{2}$ is $\left(\mathcal{J}\left(\mathfrak{m}_{x} \cdot \mathfrak{a}^{\lambda}\right), \mathcal{J}\left(\mathfrak{a}^{\lambda}\right)\right)$-regular near $x$. Suppose that $\mathrm{c}_{x}\left(\mathfrak{a}^{\lambda}\right)=0$. Then the following conditions are equivalent:

(1) The connecting homomorphism

$$
H^{1}\left(K_{\bullet}\left(a_{1}, a_{2} ;\left(\mathcal{J}\left(\mathfrak{a}^{\lambda}\right) / \mathcal{J}\left(\mathfrak{m}_{x} \cdot \mathfrak{a}^{\lambda}\right)\right)_{x}\right)\right) \rightarrow H^{2}\left(K_{\bullet}\left(a_{1}, a_{2} ; \mathcal{J}\left(\mathfrak{m}_{x} \cdot \mathfrak{a}^{\lambda}\right)_{x}\right)\right)
$$

is injective. 
(2) $H^{1}\left(K_{\bullet}\left(a_{1}, a_{2} ; \mathcal{J}\left(\mathfrak{a}^{\lambda}\right)_{x}\right)\right)=0$.

(3) $a_{1}, a_{2}$ is $\mathcal{J}\left(\mathfrak{a}^{\lambda}\right)_{x}$-regular sequence.

In particular, if one of these conditions holds, then

$$
\operatorname{depth}_{x, X} \mathcal{J}\left(\mathfrak{a}^{\lambda}\right) \geq 2 \text {. }
$$

Proof. Consider

$$
0 \longrightarrow \mathcal{J}\left(\mathfrak{m}_{x} \cdot \mathfrak{a}^{\lambda}\right) \longrightarrow \mathcal{J}\left(\mathfrak{a}^{\lambda}\right) \longrightarrow \mathcal{J}\left(\mathfrak{a}^{\lambda}\right) / \mathcal{J}\left(\mathfrak{m}_{x} \cdot \mathfrak{a}^{\lambda}\right) \longrightarrow 0 .
$$

Theorem 2.16 implies that the natural map

$$
\varphi: H^{1}\left(K_{\bullet}\left(a_{1}, a_{2} ; \mathcal{J}\left(\mathfrak{m}_{x} \cdot \mathfrak{a}^{\lambda}\right)_{x}\right)\right) \rightarrow H^{1}\left(K_{\bullet}\left(a_{1}, a_{2} ; \mathcal{J}\left(\mathfrak{a}^{\lambda}\right)_{x}\right)\right)
$$

is a zero map. Hence the equivalence of (1) and (2) follows from the long exact sequence induced from (3.1). Since $X$ is irreducible and reduced,

$$
H^{0}\left(K_{\bullet}\left(a_{1}, a_{2} ; \mathcal{J}\left(\mathfrak{a}^{\lambda}\right)_{x}\right)\right)=\left(0:\left(a_{1}, a_{2}\right)\right)_{\mathcal{J}\left(\mathfrak{a}^{\lambda}\right)_{x}}=0 .
$$

Now the equivalence of (2) and (3) follows from standard facts on Koszul complex (cf. Corollary 17.5 and Theorem 17.6 in [Eis99]).

Next, we prove Theorem 1.4 .

Proof of Theorem 1.4. Let $\mathrm{c}:=\mathrm{c}_{x}\left(\mathfrak{a}^{\lambda}\right)$. Then

$$
\operatorname{depth}_{x, X} \mathcal{J}\left(\mathfrak{m}_{x}^{c+1} \cdot \mathfrak{a}^{\lambda}\right)=1
$$

by Proposition 3.6. For any $n \geq 0$,

$$
\operatorname{depth}_{x, X} \mathcal{J}\left(\mathfrak{m}_{x}^{n} \cdot \mathfrak{a}^{\lambda}\right)=1
$$

implies $\mathrm{c}_{x}\left(\mathfrak{m}_{x}^{n} \cdot \mathfrak{a}^{\lambda}\right)=0$ by Corollary 3.8 hence

$$
\operatorname{depth}_{x, X} \mathcal{J}\left(\mathfrak{m}_{x}^{n+1} \cdot \mathfrak{a}^{\lambda}\right)=1
$$

by Proposition 3.6. The rest of the statement is clear.

\section{INTEGRAL CLOSURE AND MULTIPLIER IDEAL}

Below, we will prove a theorem that generalizes Theorem 1.7. First, we need a definition.

Definition 4.1. Let $X$ be a smooth affine variety of dimension $d \geq 2$. Let $\mathfrak{a}$ be an ideal sheaf on $X$. Let $\lambda$ be a non-negative rational number. Let $Z$ be a proper smooth subvariety of $X$ and $\mathcal{I}_{Z}$ be the ideal sheaf along $Z$. We define

$$
\mu\left(Z, \mathfrak{a}^{\lambda}\right)
$$

to be the smallest non-negative integer $c$ satisfying the following property: Let $H_{1}, \ldots, H_{c}$ be $c$ general hypersurfaces containing $Z$. Let $V=\bigcap_{i=1}^{c} H_{i}$. Then for any non-negative integer $k$ and $n$,

$$
\mathcal{I}_{Z / V}^{k} \cdot \mathcal{J}\left(\mathcal{I}_{Z / V}^{n} \cdot\left(\mathfrak{a}_{V}\right)^{\lambda}\right)
$$

is integrally closed, where $\mathcal{I}_{Z / V}$ and $\mathfrak{a}_{V}$ are the images of $\mathcal{I}_{Z}$ and $\mathfrak{a}$ in $\mathcal{O}_{V}$, respectively.

Remark 4.2. (1) Suppose that $Z$ is a divisor in $V$. Then $\mathcal{I}_{Z / V}$ is a line bundle on $V$ and $\mathcal{I}_{Z / V}^{k} \cdot \mathcal{J}\left(\mathcal{I}_{Z / V}^{n} \cdot\left(\mathfrak{a}_{V}\right)^{\lambda}\right)=\mathcal{J}\left(\mathcal{I}_{Z / V}^{k+n} \cdot\left(\mathfrak{a}_{V}\right)^{\lambda}\right)$. Hence $\mu\left(Z, \mathfrak{a}^{\lambda}\right) \leq$ $\operatorname{codim} Z-1$ by Proposition 2.19. 
(2) Even though $\mu\left(Z, \mathfrak{a}^{\lambda}\right)$ is a minimum, it does not guarantee that, for all $c>\mu\left(Z, \mathfrak{a}^{\lambda}\right), \mathcal{I}_{Z / V}^{k} \cdot \mathcal{J}\left(\mathcal{I}_{Z / V}^{n} \cdot\left(\mathfrak{a}_{V}\right)^{\lambda}\right)$ is integrally closed for any $k$ and $n$ where $V$ is the intersection of $c$ general hypersurfaces.

The following proposition gives an upper bound of $\mu\left(Z, \mathfrak{a}^{\lambda}\right)$.

Proposition 4.3. Let $X$ be a smooth affine variety of dimension $d \geq 2$. Let $\mathfrak{a}$ be an ideal sheaf on $X$ and let $\lambda$ be a non-negative rational number. Let $\bar{Z}$ be a smooth subvariety of $X$. If $\operatorname{dim} Z \leq 1$, then

$$
\mu\left(Z, \mathfrak{a}^{\lambda}\right) \leq d-2 .
$$

Proof. If $d=2$, then $\mu\left(Z, \mathfrak{a}^{\lambda}\right)=0$ by Theorem 2.18 and Proposition 2.19. So assume that $d \geq 3$. We choose an intersection $S$ of $d-2$ general hypersurfaces containing $Z$. Then

$$
\mathcal{I}_{Z / S}^{k} \cdot \mathcal{J}\left(\left(\mathfrak{a}_{S}\right)^{\lambda}\right)=\overline{\mathcal{I}_{Z / S}^{k} \cdot \mathcal{J}\left(\left(\mathfrak{a}_{S}\right)^{\lambda}\right)}
$$

by Theorem 2.18 and Proposition 2.19, Hence $\mu\left(Z, \mathfrak{a}^{\lambda}\right) \leq d-2$.

Theorem 1.7 follows from Proposition 4.3 and the following theorem.

Theorem 4.4. Let $X$ be a smooth affine variety of dimension $d \geq 2$. Let $\mathfrak{a}$ be an ideal sheaf on $X$ and let $\lambda$ be a non-negative rational number. Let $Z$ be a smooth subvariety of $X$ and $\mathcal{I}_{Z}$ be the ideal sheaf along $Z$. Let $k$ and $n$ be two non-negative integers. If $n \geq \mu\left(Z, \mathfrak{a}^{\lambda}\right)$, the ideal sheaf

$$
\mathcal{I}_{Z}^{k} \cdot \mathcal{J}\left(\mathcal{I}_{Z}^{n} \cdot \mathfrak{a}^{\lambda}\right)
$$

is integrally closed.

Proof. We use induction on $\mu:=\mu\left(Z, \mathfrak{a}^{\lambda}\right)$ and $k$. We may assume that $\mu>0$ and $k>0$. Note that $\mu>0$ implies that $d \geq 3$. Let $H$ be a general hypersurface containing $Z$. Denote by $\mathcal{I}_{Z / H}$ and $\mathfrak{a}_{H}$ the images of $\mathcal{I}_{Z}$ and $\mathfrak{a}$ in $\mathcal{O}_{H}$, respectively. Since $H$ is general, we may and do assume that

$$
\mu\left(Z,\left(\mathfrak{a}_{H}\right)^{\lambda}\right) \leq \mu-1 \leq n-1 .
$$

By Theorem 2.4 and the induction hypothesis on $\mu$, the ideal sheaf

$$
\left(\mathcal{I}_{Z}^{k} \cdot \mathcal{J}\left(\mathcal{I}_{Z}^{n} \cdot \mathfrak{a}^{\lambda}\right)\right) \cdot \mathcal{O}_{H}=\mathcal{I}_{Z / H}^{k} \cdot \mathcal{J}\left(\mathcal{I}_{Z / H}^{n-1} \cdot\left(\mathfrak{a}_{H}\right)^{\lambda}\right)
$$

is integrally closed. Since $\mathcal{I}_{Z}^{k-1} \cdot \mathcal{J}\left(\mathcal{I}_{Z}^{n} \cdot \mathfrak{a}^{\lambda}\right)$ is integrally closed by the induction hypothesis on $k, \mathcal{I}_{Z}^{k} \cdot \mathcal{J}\left(\mathcal{I}_{Z}^{n} \cdot \mathfrak{a}^{\lambda}\right)$ is also integrally closed by Proposition 2.17 .

We obtain the following

Corollary 4.5. Let $X$ be a smooth affine variety of dimension $d \geq 2$. Let $\mathfrak{a}$ be an ideal sheaf on $X$ and let $\lambda$ be a non-negative rational number. Let $C \subset X$ be a smooth curve on $X$ and $\mathcal{I}_{C}$ be the ideal sheaf along $C$. Let $k$ and $n$ be two non-negative integers. If $n \geq d-2$, the ideal sheaf

$$
\mathcal{I}_{C}^{k} \cdot \mathcal{J}\left(\mathcal{I}_{C}^{n} \cdot \mathfrak{a}^{\lambda}\right)
$$

is integrally closed.

The following theorem generalizes Theorem 1.8 slightly. 
Theorem 4.6. Let $X$ be a smooth affine variety of dimension $d \geq 2$. Let $\mathfrak{a}$ be an ideal sheaf on $X$ and let $\lambda$ be a non-negative rational number. Let $Z$ be a smooth subvariety of $X$ with $\operatorname{codim} Z \geq 2$. Let $1 \leq c \leq \operatorname{codim} Z-1$ and let $H_{1}, \ldots, H_{c}$ be $c$ general hypersurfaces containing $Z$. Let $V=\bigcap_{i=1}^{c} H_{i}$. Then for any integer $k \geq c$, the ideal sheaf

$$
\mathcal{I}_{V} \otimes \mathcal{J}\left(\mathcal{I}_{Z}^{k-1} \cdot \mathfrak{a}^{\lambda}\right)
$$

is integrally closed, where $\mathcal{I}_{Z}$ and $\mathcal{I}_{V}$ are the ideal sheaves along $Z$ and $V$, respectively.

Proof. Since $V$ is general, $\mathcal{I}_{V}$ is reduced, hence integrally closed. Then since the intersection of integrally closed ideal sheaves are again integrally closed, the proposition follows from Proposition 2.17 and Corollary 2.5.

Proof of Theorem 1.6. Theorem 1.4 and our assumption imply that

$$
\mathrm{c}_{x}\left(\mathfrak{a}^{\lambda}\right) \geq d-2 .
$$

Hence

$$
\mathcal{J}\left(\mathfrak{m}_{x}^{d-2} \cdot \mathfrak{a}^{\lambda}\right)=\mathcal{J}\left(\mathfrak{a}^{\lambda}\right) .
$$

Now the theorem follows from Theorem 1.7

\section{ACKNOWLEDGMENT}

The author would like to thank the referee for fixing a gap and for many suggestions which helped to improve the presentation of the paper.

\section{REFERENCES}

[EM06] Lawrence Ein and Mircea Mustata, Invariants of singularities of pairs, International Congress of Mathematicians. Vol. II, 583-602, Eur. Math. Soc., Zurich, 2006. MR2275611 (2007m:14050)

[Eis99] David Eisenbud, Commutative algebra, with a view toward algebraic geometry, 3rd ed., Graduate Text in Mathematics, Springer-Verlag, 1999. MR1322960 (97a:13001)

[HY03] Nobuo Hara and Ken-ichi Yoshida, A generalization of tight closure and multiplier ideals, Trans. Amer. Math. Soc. 355 (2003), 3143-3174. MR1974679 (2004i:13003)

[HH99] Melvin Hochster and Craig Huneke, Tight closure in equal characteristic zero, Preprint, 1999. MR:1015524 (91f:13022)

[Hun86] Craig Huneke, The primary components of and integral closure of ideals in 3-dimensional regular local rings, Math. Ann. 275 (1986), 617-635. MR859334 (87k:13038)

[Laz00] Robert Lazarsfeld, Positivity in algebraic geometry II, 1st ed., Ergebnisse der Mathematik und ihrer Grenzgebiete 3. Folge, Springer-Verlag, 2000. MR2095472 (2005k:14001b)

[LL06] Robert Lazarsfeld and Kyungyong Lee, Local syzygies of multiplier ideals, Invent. Math. 167 (2007), 409-418. MR2270459 (2007h:13021)

[Lee06] Seunghun Lee, Filtrations and local syzygies of multiplier ideals, J. Algebra 315 (2007), 629-639. MR2351883

[Lip69] Joseph Lipman, Rational singularities, with applications to algebraic surfaces and unique factorization, Inst. Hautes Etudes Sci. Publ. Math. 36 (1969), 195-279. MR0276239 (43:1986)

[Tak04] Shunsuke Takagi, An interpretation of multiplier ideals via tight closure, J. Algebraic Geom. 13 (2004), 393-415. MR.2047704(2005c:13002)

[ZS75] Oscar Zariski and Pierre Samuel, Commutative algebra II, Graduate Texts in Mathematics, Springer-Verlag, 1975.

Department of Mathematics, Konkuk University, Kwangun-Gu Hwayang-Dong 1, SEOUl 143-701, Korea

E-mail address: mbrs@konkuk.ac.kr 